\title{
Biomímesis, inspiración natural para la innovación
}

\section{Biomimesis, a natural inspiration for innovation}

\author{
Ruth Zorayda Osorio \\ Gestor de Proyectos, Red Tecnoparque SENA Nodo Medellín - Grupo GIGAT, Servicio Nacional de Aprendizaje SENA, \\ Medellín-Colombia rzosorio@misena.edu.co \\ Editorial
}

En el mundo, las tendencias actuales de innovación suscitan la búsqueda de nuevas alternativas y herramientas de desarrollo [1]. Biomímesis es un término poco conocido, solo algunas personas se hallan familiarizadas con esta palabra. Esta disciplina, también conocida como biomimética, aparece cada vez más influyente en los campos de las ciencias sociales, naturales, humanas, formales y aplicadas, y puede decirse que ha tomado una gran fuerza en la última década. La biomímesis hace referencia al estudio de la naturaleza y su emulación en todas las áreas sociales e industriales [2], [3]; el término "biomimética" fue acuñado por primera vez en la década de 1990 por Janine Benyus (1997) en su libro "Biomimicry: Innovation Inspired by Nature" [4]. Su metodología consiste en una detallada comprensión de los principios fundamentales de la naturaleza, en sus sistemas y procesos biológicos, interacciones, habilidades de supervivencia, entre muchas otras características, para adaptarlos posteriormente a la solución de desafíos específicos en diferentes áreas [5].

En los últimos 5 años se han escrito alrededor de 761 artículos relacionados con biomímesis según consulta de la base de datos Scopus, donde el área de ingeniería posee un $22.2 \%$, el área de materiales un $15.6 \%$ y las ciencias sociales $2.9 \%$ con respecto al total. Además, es Estados Unidos quien se posiciona como el país que más aporta en publicaciones relacionadas, con un total de 240 documentos. ISO/TC 266 es el comité de estándares en biomimética, y cuenta con 6 publicaciones hasta el momento [6]. Además, la revista Forbes ha sugerido que esta disciplina es una de las cinco tendencias tecnológicas que pueden impulsar el éxito de las carreras empresariales [7]. La firma Fermian Businnes ha estimado que para el año 2030 la biomimética habrá contribuido con $\$ 1.6$ trillones de dólares al PIB mundial a partir de sus diferentes desarrollos o las actividades derivadas de ella [8]. No se puede negar que solo con conocer estos datos, parece una disciplina muy atractiva para todos aquellos inquietos por los desarrollos innovadores o por los amantes a las soluciones que no generen impactos negativos sobre la naturaleza y sus recursos, o mejor aún, sobre todos aquellos empresarios que requieren soluciones efectivas y eficaces para sus retos.

En el mundo empresarial, donde siempre se buscan nuevas soluciones a los problemas o necesidades para mejorar o crear nuevos servicios o productos que se hallen acorde a las nuevas políticas o tendencias sociales o ingenieriles, la biomímesis representa un terreno fértil como herramienta para la innovación, ya que esta tendencia es un área emergente llena de oportunidades para encontrar soluciones rentables, sustentables y sostenibles. Es toda una inspiración para resolver los desafíos que aún nos apremian, transformado la forma que tenemos de mirar las cosas. Como ejemplo de ello se tienen diferentes productos y servicios basados en principios biomiméticos de empresas como: Sharklet [9] o Columbia Forest Products [10], por mencionar dos de ellas.

Esta especie de revolución a partir de la biomímesis parte de un concepto simple, si se quiere, pues si la naturaleza ha desarrollado por cerca de 3.8 millones de años estrategias, habilidades, sistemas de cooperación y trabajo, esquemas tácticos y de sostenibilidad que le han permitido permanecer en el tiempo siempre renovándose, ¿por qué no pensar en ella como fuente de inspiración? Todos estos años de "investigación" e "innovación" por parte de la naturaleza permitirán intervenir en sistemas, procesos, diseños, comunidades, productos y servicios, al emular sus propiedades únicas.

Citar como:

R. Z. Osorio. “Biomímesis, inspiración natural para la innovación” Revista CINTEX, Vol. 24(2), pp. $10-11.2019$. 
Con todo lo anterior aún me pregunto porque solo algunas personas en Colombia trabajan esta disciplina, de hecho, existen unas pocas asociaciones y universidades que han estimulado investigaciones y desarrollos en el tema. Pero aún falta, por eso quiero dejar la pregunta sobre la mesa para todos aquellos investigadores y empresarios que desean alcanzar desarrollos sostenibles: ¿ de qué manera puedes incluir los principios de la naturaleza en el desarrollo e innovación de tus productos y servicios?

\section{REFERENCIAS}

[1] J. A. Londoño-Gallego, S. M. Velásquez Restrepo, M. E. Villa Rodríguez, F. D. J. Franco Cuartas, y N. E. Viana-Rúa, «Identificación De Tipos, Modelos Y Mecanismos De Transferencia Tecnológica Que Apalancan La Innovación», Rev. CINTEX, vol. 23, n. 2, pp. 13-23, dic. 2018.

[2] E. Mejía, L. Osorno, y J. Ospina, «Microorganismos Hierro-Azufre Oxidantes Una Alternativa Biotecnológica», Rev. CINTEX, vol. 19, pp. 63-77, 2014.

[3] H. D. Ortiz Alzate, L. G. Muñoz Marín, J. Cardeño Espinosa, y N. C. Alzate Osorno, «lmpacto del uso de objetos interactivos de aprendizaje en la apropiación de conocimiento y su contribución en el desarrollo de competencias matemáticas: un resultado de experiencia de investigación», Rev. CINTEX, vol. 21, n. ${ }^{\circ}$, pp. 7188, jun. 2016.

[4] J. M. Benyus, Biomimicry: innovation inspired by nature, Repr. New York, N.Y: Harper Perennial, 2008.

[5] E. Lurie-Luke, «Product and technology innovation: What can biomimicry inspire?», Biotechnol. Adv., vol. 32, n. ${ }^{\circ}$ 8, pp. 1494-1505, dic. 2014, doi: 10.1016/j.biotechadv.2014.10.002.

[6] N. Hoeller y F. A. Salustri, «Advancing biomimetic materials through ISO standards», Bioinspired Biomim. Nanobiomaterials, vol. 5, n. ${ }^{\circ} 4$, pp. 171-175, dic. 2016, doi: 10.1680/jbibn.16.00011.

[7] R. Bagley, «Five Tech Trends That Can Drive Company Success», Forbes, 01-abr-2014.

[8] S. A. Fernhaber y A. Y. Stark, «Biomimicry: New insights for entrepreneurship scholarship», J. Bus. Ventur. Insights, vol. 12, p. e00137, nov. 2019, doi: 10.1016/j.jbvi.2019.e00137.

[9] A. R. Karabetça, «Nature Inspired Architectural Designs: Using Biomimicry as a Design Strategy», en International Conference on New Trends in Architecture and Interior Design. Proceeding Book, 2015, pp. 143151.

[10] E. B. Kennedy, «Biomimicry: Design by Analogy to Biology», Res.-Technol. Manag., vol. 60, n. ${ }^{\circ}$ 6, pp. 51-56, nov. 2017, doi: 10.1080/08956308.2017.1373052. 